\title{
Análisis de los eventos adversos en el área de rehabilitación oral de la Facultad de Odontología de la Pontificia Universidad Javeriana Bogotá
}

Analysis of Adverse Events in the Oral Rehabilitation Area at the Pontificia Universidad Javeriana Dentistry School in Bogotá

Wendy Pérez Gómez

Práctica privada. Bogotá, Colombia

wendyprezg@gmail.com

Alejandra Maria Pita Bejarano

Práctica privada, Guayaquil, Ecuador

alejandrapitabejarano@gmail.com

Carlos Alberto Ramos Vargas

Pontificia Universidad Javeriana, Bogotá, Colombia,

Colombia

carlos.ramos@javeriana.edu.co

Julian González Moncada

Pontificia Universidad Javeriana, Bogotá, Colombia,

Colombia

juliana.gonzalez@javeriana.edu.co

Édgar Humberto Güiza Cristancho

Pontificia Universidad Javeriana, Bogotá, Colombia,

Colombia

eguiza@javeriana.edu.co

Adriana Rodriguez Ciódaro

Pontificia Universidad Javeriana, Bogotá, Colombia,

Colombia

arodrig@javeriana.edu.co

\section{Resumen:}

Antecedentes: Uno de los mecanismos para garantizar la calidad de la atención en salud es el análisis de eventos adversos en los tratamientos. El área de la rehabilitación oral es una de las más propensas, dada la complejidad de sus procedimientos. Objetivo: Analizar los eventos adversos que se presentaron en la clínica del posgrado de rehabilitación oral de la Facultad de Odontología de la Pontificia Universidad Javeriana de Bogotá durante 2013. Métodos: De 595 historias clínicas correspondientes a todos los pacientes que finalizaron su tratamiento en 2013, 590 cumplieron con el requisito de estar firmadas por el paciente y por el profesor. A partir de las historias que contenían algún reporte de evento adverso, se registraron los datos demográficos de los pacientes, los tipos de eventos adversos reportados, las posibles causas que su ocurrencia y si eran o no prevenibles. Resultados: En 36 (6,1 \%) de las historias clínicas analizadas se encontró algún reporte de evento adverso. El evento más frecuente fue la pérdida de la restauración (42\%). La causa más frecuente fue la fractura completa de la restauración (19\%). El $58 \%$ se consideró evento adverso prevenible. En el 61,1 \% de los casos fueron prótesis fijas dentorretenidas. Conclusiones: La frecuencia de eventos adversos reportada en las historias clínicas de pacientes atendidos en el área de rehabilitación es baja. Teniendo en cuenta de condición de prevenible de estos casos, es importante registrarlos y analizarlos para asegurar la calidad en la atención de los pacientes.

Palabras clave: complicaciones, evento adverso, iatrogenia, daños, prostodoncia, rehabilitación oral, seguridad del paciente.

Áreas temáticas: administración en salud; odontología; prostodoncia; rehabilitación oral 


\begin{abstract}
:
Background: One of the mechanisms that ensures the health care quality is the analysis of adverse events in the treatment. The oral rehabilitation area is among the more likely because of the complex procedures involved. Objective: To analyze the adverse events that occurred in the clinic of the oral rehabilitation graduate program at the Pontificia Universidad Javeriana Dentistry School in Bogotá during 2013. Methods: Out of 595 medical records including all the patients who completed their treatment in 2013, 590 fulfilled the requirement of being signed both by the patient and the teacher. Based on the medical records that reported any kind of adverse event, the patient information regarding demographic data, type of reported adverse event, potential causes for their occurrence, and whether they were preventable was gathered. Results: In 36 (6.1\%) of the examined medical records, at least one report of adverse event was found. The most frequently found event was the restoration loss (42\%). The most frequently found cause was the full fracture of the restoration (19\%). Fifty-eight (58\%) of the adverse events were preventable. Sixty-one $(61.1 \%)$ of the cases were retained fixed dental prostheses. Conclusions: The frequency of adverse events reported in the medical records of patients treated in the rehabilitation area is low. Considering that the cases are preventable, it is important to record and examine these cases in order to ensure the health care quality for the patients.
\end{abstract} Keywords: complications, adverse event, iatrogeny, damages, prosthodontics, oral rehabilitation, patient safety.

Thematic fields: dentistry; health administration; oral rehabilitation; prosthodontics

\title{
INTRODUCCIÓN
}

La poca seguridad para un paciente es un inconveniente grave de salud pública en todo el mundo. Afecta a personas tanto de países desarrollados como de aquellos en vías de desarrollo y ocurre en todos los ámbitos de atención. Por tal razón, la Organización Mundial de la Salud decidió formar una alianza con otros líderes mundiales de salud, a fin de reducir el número de enfermedades, lesiones y muertes que ocurren como consecuencia de errores en la atención en salud $(1,2)$.

En Colombia se han establecido políticas públicas sobre el tema que siguen los lineamientos de la Organización Mundial de la Salud. En la Resolución 1446 del 2006, el Ministerio de Protección Social de la República de Colombia define el término evento adverso como:

Las lesiones o complicaciones involuntarias que ocurren durante la atención en salud, las cuales son más atribuibles a esta que a la enfermedad subyacente y que pueden conducir a la muerte, la incapacidad o al deterioro en el estado de salud del paciente, a la demora del alta, la prolongación del tiempo de estancia y al incremento de los costos de la no calidad. (3)

En 2008, se formuló la Política de Seguridad, cuyo objetivo es prevenir la ocurrencia de situaciones que afecten la seguridad del paciente, reducir y, de ser posible, eliminar la ocurrencia de eventos adversos para contar con instituciones que eviten atenciones inseguras (4).

En 2011, se estableció el Sistema Obligatorio de Garantía de Calidad de la Atención en Salud, con el objetivo de minimizar los riesgos en el proceso de atención de salud y prevenir la ocurrencia de situaciones que afecten la seguridad del paciente. Además, el sistema busca que las instituciones brinden seguridad a los pacientes durante su atención médica, por medio del reporte obligatorio de los indicadores de calidad y monitorización permanente del desempeño de la gestión de la vigilancia de los eventos adversos. Los componentes de este sistema son: 1) el sistema único de habilitación, 2) la auditoría para el mejoramiento de la calidad de la atención en salud, 3) el sistema único de acreditación y 4) el sistema de información para la calidad (5).

La Resolución 2003 de 2014 estableció los criterios y las condiciones con las cuales deben contar todos los servicios de salud para garantizar estándares mínimos de cumplimiento obligatorio y asegurar que los servicios se presten en condiciones que minimicen los riesgos de seguridad para los pacientes. Uno de los procesos prioritarios es que todas las instituciones están obligadas a contar con un programa de seguridad del paciente que provea una adecuada caja de herramientas para la identificación y la gestión de eventos adversos, e incluir una política formal de seguridad del paciente acorde con los lineamientos para la implementación de la política de seguridad del paciente en la República de Colombia (6).

La estrategia de seguridad busca que las instituciones y los profesionales sean cada vez más hábiles para identificar los eventos adversos más frecuentes que suceden durante el proceso de atención y aprendan 
a gestionarlos y prevenirlos, instaurando progresivamente la cultura de seguridad del paciente, que se ha trasformado en uno de los objetivos estratégicos de las instituciones. Hace parte fundamental de esta planeación conocer los eventos adversos que ocurren en cada servicio de salud ofrecido. Una de las investigaciones más grandes reportadas al respecto la realizaron Perea y colaboradores (7), en la cual se revisaron los eventos adversos odontológicos reportados por el Observatorio Español para la Seguridad del Paciente Odontológico entre 2000 y 2010. Encontraron que los tratamientos de implantología oral, endodoncia y cirugía oral mostraron mayores frecuencias de eventos adversos en porcentajes del 25,5\%, 20,7 $\%$ y 20,4 \%, respectivamente. Además, el 44,3\% de los eventos adversos reportados correspondió a eventos adversos prevenibles.

En Colombia poco se ha informado sobre eventos adversos en odontología en instituciones particulares o públicas que se encuentran vinculadas a la prestación de servicios de salud dentro del Sistema de Seguridad Social en Salud. Tampoco hay estudios de eventos presentados en la consulta clínica de odontólogos que trabajan de forma independiente. Ello puede llevar a inferir que, aunque la seguridad del paciente no es tema nuevo en Colombia, aún falta implementar una gestión clínica que busque mejorar la seguridad del paciente en la consulta odontológica, mediante el diseño de procesos seguros para evitar eventos adversos prevenibles.

Incluso así, en la Facultad de Odontología de la Pontificia Universidad Javeriana de Bogotá este tema ha tomado importancia desde la calidad de la atención en el modelo de atención de docencia de servicio. A la fecha, se han publicado resultados desde las especialidades de endodoncia (8) y periodoncia (9), con el propósito de determinar la frecuencia y la naturaleza de los eventos adversos, utilizando los reportes en las historias clínicas y mediante el análisis de los eventos adversos reportados a la Dirección de Clínicas de la Facultad (10).

En ambas metodologías se encontró una baja frecuencia de reportes de eventos adversos, en su mayoría de naturaleza prevenible. Para completar esta información, es importante realizar estudios en otras especialidades como rehabilitación oral, odontopediatría y ortodoncia, con el fin de que, en conjunto, los resultados de tales estudios contribuyan a proponer un modelo de vigilancia e informe de eventos adversos en las facultades de odontología, que oriente a docentes y estudiantes hacia una cultura corporativa de seguridad mediante actividades efectivas que incentiven notificar los eventos adversos entre todo el personal académico, estudiantil y administrativo. Por tal razón, se planteó como objetivo de esta investigación analizar los eventos adversos que se presentaron en la clínica del posgrado de Rehabilitación Oral de la Facultad de Odontología de la Pontificia Universidad Javeriana de Bogotá durante 2013, a efectos de darles continuidad a las investigaciones realizadas en años anteriores y en otras especialidades dentro del modelo académico de prácticas formativas en las especialidades odontológicas.

La importancia de este trabajo en la docencia es la integración técnico-científica en la enseñanza de cada estudiante, mediante un proceso de planificación académica, administrativa e investigativa que promueva la proactividad del estudiante y sus docentes en la gestión y reporte de los eventos adversos presentados, así como recalcar la importancia de la calidad de los registros clínicos. Esto permitirá mejorar la atención de los usuarios que asisten a instituciones académicas en busca de tratamiento bajo el modelo docente-asistencial. Esta formación también contribuye a que los futuros profesionales en odontología y sus especialidades, en el momento de vincularse a un campo laboral, cuenten con las competencias administrativas que los hagan más competitivos y puedan desempeñarse exitosamente usando un modelo de atención fundamentado en la seguridad del paciente.

En consecuencia, y según lo planteado, se caracterizaron los eventos adversos que se presentaron en los procedimientos realizados en el área de Rehabilitación Oral durante 2013 y se determinaron si estos eran prevenibles. La pregunta de investigación fue: ¿cuál es la frecuencia y cómo se caracterizan los eventos adversos que se presentaron en el área de Rehabilitación Oral en las clínicas de posgrado en la Facultad de Odontología de la Pontificia Universidad Javeriana de Bogotá? 


\section{MATERIALES Y MÉTODOS}

Se realizó un estudio descriptivo de corte transversal, el cual recibió aval del Comité de Investigación y Ética de la Facultad de Odontología de la Pontificia Universidad Javeriana. Se incluyeron todas las historias clínicas de pacientes quienes finalizaron su tratamiento durante 2013 en el posgrado de Rehabilitación Oral. Se excluyeron las historias clínicas que no tuvieran la aprobación del paciente o del profesor.

En total se revisaron 595 historias clínicas de las cuales 5 no cumplieron con los criterios de inclusión. A partir de las historias que contenían algún evento adverso, se registró la información demográfica de los pacientes, los eventos adversos informados, las posibles causas de tales eventos y su clasificación como prevenibles o no prevenibles. Adicionalmente, se registró el tipo de prótesis asociada al evento ocurrido. Los criterios que se tuvieron en cuenta para determinar si un evento adverso era o no prevenible se basaron en la definición de los lineamientos para la implementación de la política de seguridad del paciente en la República de Colombia (4). Ante la escasa literatura relacionada con los eventos adversos en rehabilitación oral, se analizaron algunos artículos que reportaban fallas o fracasos en los procedimientos relacionados con prostodoncia y se definió cuáles podrían ser clasificados como eventos adversos al usar la definición establecida por el Ministerio de Salud en Colombia $(3,11,12,13,14,15,16)$.

El procesamiento y la recolección de la información se realizó mediante el programa SPSS versión 15 . Se utilizó la prueba de chi-cuadrado para analizar la relación entre la variable edad y la presentación del evento adverso $(\alpha=0,05)$.

\section{RESULTADOS}

De las 590 historias clínicas revisadas, 395 (67\%) correspondieron a pacientes mujeres. En 36 historias clínicas $(6,1 \%)$ se informaba algún tipo de evento adverso, la mayoría (72\%) en historias clínicas de mujeres. Sin embargo, en el análisis bivariado no se encontró relación estadísticamente significativa de la presentación de eventos adversos con el sexo de los pacientes $(\mathrm{p}>0,05)$.

Se detectaron cinco tipos de eventos adversos. Como se muestra en la tabla 1, la pérdida de la restauración fue el más frecuente (42\%) y la causa más encontrada fue la fractura completa de la restauración en un $19 \%$. 
TABLA 1

Tipos y causas de los eventos adversos registrados en las historias clínicas

\begin{tabular}{lcc}
\hline Tipo de evento adverso & Número & Porcentaje \\
\hline Pérdida de la restauración & 15 & 42 \\
Pérdida dental causada por la rehabilitación oral & 13 & 36 \\
Pérdida del implante & 4 & 11 \\
Caries residual causada por la rehabilitación oral & 3 & 8 \\
Pérdida de tejido duro o blando causado por la & 1 & 3 \\
rehabilitación oral & & \\
\hline Causas de los eventos adversos presentados & Número & Porcentaje \\
\hline Fractura completa de la restauración & 7 & 19 \\
Fractura del diente pilar & 6 & 17 \\
Estallido radicular & 6 & 17 \\
Delaminación de la porcelana & 5 & 14 \\
Fracaso del implante & 4 & 11 \\
Descementación de la restauración & 4 & 11 \\
Desadaptación de la restauración & 2 & 5 \\
Fractura del núcleo colado & 1 & 3 \\
Pérdida de estabilidad, soporte y retención & 1 & 3 \\
\hline
\end{tabular}

Para determinar si el evento era o no prevenible, adicional a su naturaleza, se analizó la causa asociada a su ocurrencia. Se encontró que, de los 36 eventos adversos registrados, 21 (58 \%) se consideraron prevenibles. Con respecto al tipo de prótesis, se encontró que se presentaron más eventos adversos en prótesis fi jas dentorretenidas (figura 1). Esto ocurrió en un 61,1\% de los casos.

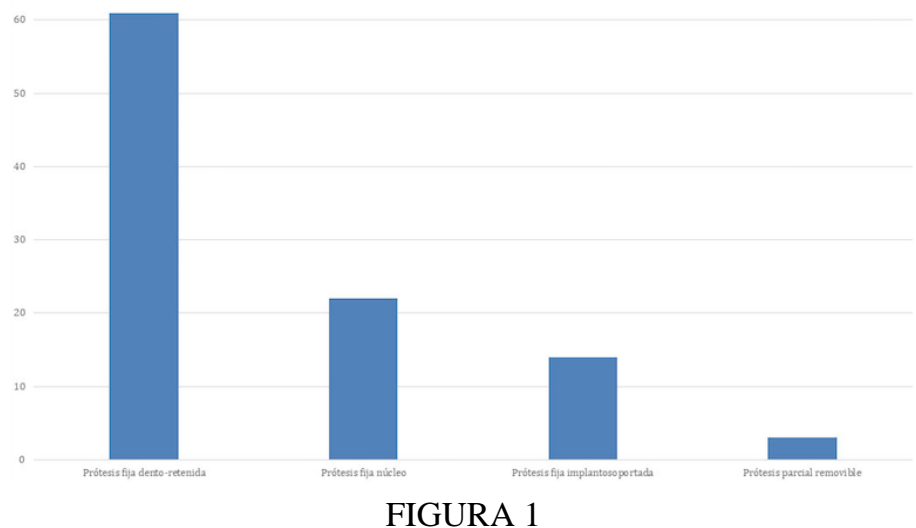

Porcentaje de las prótesis en las que se presentaron los eventos adversos analizados

\section{DISCUSIÓN}

La seguridad del paciente desempeña un papel relevante en la prestación de los servicios de salud, debido al impacto económico, la alteración en la calidad de vida y los sobrecostos que se pueden generar en el Sistema de Seguridad Social en Salud. Esta preocupación es fuerte en las prácticas clínicas formativas, por la necesidad de alcanzar altos índices de calidad, representados en la formación del estudiante y en la atención de los pacientes. De ahí que sea importante para las instituciones educativas identificar las oportunidades de mejora, caracterizando y previniendo eventos adversos.

Los pacientes de esta investigación fueron atendidos por estudiantes del posgrado de Rehabilitación Oral, quienes se encontraban en un proceso de aprendizaje, en un contexto diferente al de una práctica profesional, lo que supondría un mayor riesgo de presentarse errores en la atención. Sin embargo, el estar regulados por un modelo de atención de docencia-servicio, representa una ventaja, dado que todos los procedimientos clínicos 
deben ser validados por el docente antes de su realización, deben cumplir con los protocolos y guías de manejo clínico institucionales y ser parte del proceso de evaluación académica. Finalmente, todos los estudiantes tienen la obligación de notificar los eventos adversos que sucedan.

La falta de reporte o el reporte incompleto de los eventos adversos en el área odontológica puede llevar a problemas graves en la atención en salud, pues no permite que se detecten alteraciones en el servicio, que en muchos casos podrían ser prevenibles. La falta de información durante la elaboración de la historia clínica puede deberse al desconocimiento del evento, a mala praxis o a temor por parte del odontólogo a que se afecte la percepción de calidad de su atención. La falta de reporte podría llevar a problemas graves de salud e, incluso, a la muerte del paciente (7).

En esta investigación se evaluaron 590 historias clínicas con la intención de identificar registros de eventos adversos en el área de rehabilitación oral y analizar sus posibles causas. En este proceso se enfrentaron algunas dificultades, porque no siempre se había registrado la descripción completa del evento o no se encontraron ayudas como radiografías o fotografías clínicas que permitieran analizar lo sucedido. En la literatura sobre el tema poco se ha dado parte de ello, por lo que se considera mundialmente que la falta de reportes de eventos adversos es un problema bastante común en el área odontológica, debido a que los odontólogos estiman que los incidentes ocurridos durante la práctica no representan riesgos graves para el paciente. Además, como la mayoría de estos problemas se puede resolver durante el curso del tratamiento, raramente el paciente recurre a instancias legales (7).

Al comparar los resultados de este estudio con los de otros trabajos publicados, se observan diferencias en el número de eventos adversos informados en el área de rehabilitación oral, lo cual depende de la metodología utilizada y del origen de la información. En el estudio de Perea-Pérez y colaboradores (7), en el que se analizaron 4149 reclamaciones legales en odontología durante un periodo de 10 años, se encontraron 415 eventos adversos, $52(1,3 \%)$ relacionados con tratamientos prostodónticos. Al comparar ambos estudios, se observa en primera instancia la definición de evento adverso. En el estudio de Perea-Pérez y colaboradores se consideraron el error, la complicación y el accidente como tipos de eventos adversos, en tanto que esta investigación acogió los lineamientos para implementación de la política de seguridad del paciente en la República de Colombia, que define el evento adverso como el resultado de una atención en salud que de manera no intencional produjo daño (4).

Otro aspecto relevante es la gravedad del daño causado, dado que los eventos adversos reportados en el estudio de Perea-Pérez y colaboradores (7) involucraban reclamaciones legales registradas en el Observatorio para la Seguridad Dental del Paciente en España, que causaron en su mayoría daños graves en el paciente, generaron hospitalizaciones y solo un 3,4 \% se resolvieron en el mismo centro. En el presente estudio, todos los eventos adversos se trataron en la misma institución, bien fuera repitiendo o cambiando el plan de tratamiento.

En un estudio realizado por Huertas y colaboradores (10), en el que se analizaron las quejas que llegan a la Dirección de Clínicas de la Facultad de Odontología de la Pontificia Universidad Javeriana de Bogotá, donde se realizó el presente estudio, solo se encontró una frecuencia de eventos adversos del 0,26\%. De estos, la mayoría (62\%) correspondió a procedimientos relacionados con el área de rehabilitación oral.

En atención a la problemática expuesta, según lo presentado en la literatura, existen protocolos que deben seguirse para prevenir fracasos en los tratamientos realizados. Dichos fracasos se pueden generar por errores en el manejo de los biomateriales odontológicos, durante los procesos de laboratorio, por falta de conocimiento y dificultad en los procedimientos durante la práctica clínica en las situaciones particulares de cada paciente (17).

Este trabajo se enfoca en las causas de los eventos adversos, porque en esta etapa se pueden establecer parámetros de prevención y control y así prevenir la ocurrencia. En la literatura actual se encuentra cómo el poco control de la sangre y saliva durante los procesos de cementación; los agente cementantes (18) o de laboratorio, como factores ambientales (humedad) (11); errores técnicos durante la fabricación (12,13); el 
número de cocciones (14); la calidad de los materiales utilizados (19), o el conocimiento del operador y el técnico dental pueden llevar a fracasos en los tratamientos odontológicos. Adicionalmente, al conocer las causas de los eventos adversos se determina en qué casos se pueden mejorar los procesos de atención y, de esta forma, se evita o minimiza la ocurrencia de eventos adversos.

Pocos estudios se encuentran en la literatura en los cuales se relacionen los tipos de prótesis con los eventos adversos. En esta investigación se encontró que el $61 \%$ de los eventos se presentaron en prótesis fija dentorretenida. Las posibles causas incluyen una combinación de variables del paciente, del rehabilitador y del laboratorio o técnico dental. Teniendo en cuenta lo anterior, es importante tener mayor control de todos los procedimientos clínicos y de laboratorio. El profesional de la salud debe tratar de minimizar los errores durante los tratamientos y tener conocimientos de ellos para optimizar los procesos de laboratorio, evitando que se presenten eventos adversos prevenibles.

\section{CONCLUSIONES}

La frecuencia de eventos adversos en el área de rehabilitación oral fue baja. El evento adverso que se encontró con mayor frecuencia fue la pérdida de la restauración y la pérdida del diente causado por la rehabilitación oral, evento prevenible en la mayoría de los casos revisados.

\section{RECOMENDACIONES}

Diseñar estrategias para mejorar el registro de los eventos adversos, que incluyan fotos y radiografías que documenten el momento en que se presentó el evento para contar con mejores herramientas de análisis y minimizar su ocurrencia.

Incluir dentro de la historia clínica los pasos de laboratorio para detectar si el origen del evento es clínico o del laboratorio.

\section{AGRADECIMIENTOS}

Este trabajo fue avalado por el Comité de Investigación y Ética de la Facultad de Odontología de la Pontificia Universidad Javeriana, según consta en el acta 013 del 2012.

\section{Referencias}

1. Organización Mundial de la Salud (OMS). La alianza mundial para la seguridad del paciente. La Investigación en Seguridad del Paciente. Mayor Conocimiento para una atención más segura [internet]. Ginebra, Suiza: OMS; 2008. Disponible en: https://www.who.int/patientsafety/information_centre/documents/ps_research_broch ure_es.pdf

2. García-Barbero M. La alianza mundial para la seguridad del paciente [internet]. Valencia, España: Consejo de Enfermería de la Comunidad Valenciana; 2007. Disponible en: https://www.portalcenova.es/es/grupos/biolog icos/pacientes/alianza_mundial_seguridad_paciente.pdf

3. República de Colombia. Resolución 1446 del 8 de mayo de 2006. Sistema de Información para la Calidad y se adoptan los indicadores de monitoría del Sistema Obligatorio de Garantía de Calidad de la Atención en Salud [internet]. Bogotá, Colombia: Ministerio de Salud y Protección Social; 2008. Disponible en: https://www.minsalud.gov.co/Normatividad_Nuevo/RESOLUCI\%C3\%93N\%201446\%20DE\%202006 \%20-\%20ANEXO\%20T\%C3\%89CNICO.pdf 
4. Ministerio de Salud y Protección Social. Lineamientos para la implementación de la política de seguridad del paciente en la República de Colombia [internet]. Bogotá, Colombia: el Ministerio; 2008. Disponible en: https://www.a creditacionensalud.org.co/catalogodocsLineamientos\%20politica\%20seguridad\%20paciente.pdf

5. República de Colombia. Ley 1438, que establece el Sistema Obligatorio de Garantía de Calidad de la Atención en Salud. Bogotá, Colombia: Ministerio de Salud y Protección Social; 2011.

6. República de Colombia. Resolución 00002003 [internet]. Bogotá, Colombia: Ministerio de Salud y Protección Social; 2014. Disponible en: https://www.minsalud.gov.co/Normatividad_Nuevo/Resoluci\%C3\%B3n\%2020 03\%20de\%202014.pdf

7. Perea-Pérez B, Labajo-González E, Santiago-Sáez A, Albarrán-Juan E, Villa-Vigil A. Analysis of 415 adverse events in dental practice in Spain from 2000 to 2010. Med Oral Patol Oral Cir Bucal. 2014 Sep; 19(5): 500-5. https: //doi.org/10.4317/medoral.19601

8. Tafur Gallego M, Camacho Alonso L, Mejía Morales S, González Moncada J, Huertas de Hoyos M. Frecuencia de eventos adversos de la terapia endodóntica y seguimiento de pacientes atendidos en el Posgrado de Endodoncia de la Pontificia Universidad Javeriana (2007-2008). Univ Odontol. 2014 Jul-Dic; 33(71): 98-107. https://dx.d oi.org/1011144/Javeriana.uo33-71.feat

9. Tafur DJ, Ramírez GP, Cárdenas CA, Serrano JJ, Sarralde AL, Camacho SP, Rodríguez A, González J. Características y prevalencia de los eventos adversos presentados en la Clínica del Posgrado de Periodoncia de la Facultad de Odontología de la Pontificia Universidad Javeriana durante el período 2011-2012. Univ Odontol. 2016 Jul-Dic; 35(75). https://dx.doi.org/10.11144/Javeriana.uo35-75.cpea

10. Huertas MF, González J, Camacho S, Sarralde AL, Rodríguez A. Analysis of the adverse events reported to the office of the clinical director at a dental school in Bogotá, Colombia. Acta Odontol Latinoam. 2017 Apr; 30(1): 19-25.

11. Ozcan M. Fracture reasons in ceramic-fused-to-metal restorations. J Oral Rehabil. 2003 Mar; 30(3): 265-9. http s://doi.org/10.1046/j.1365-2842.2003.01038.x

12. Oram DA, Davies EH, Cruickshank-Boyd DW. Fracture of ceramic and metallic ceramic cylinders. J Prosthet Dent. 1984 Aug; 52(2): 221-30.

13. Øilo G. Flexural strength and internal defect of some dental porcelain. Acta Odontol Scand. 1988 Oct; 46(5): 313-22. https://doi.org/10.3109/00016358809004782

14. Mackert JR, Williams AL. Microcracks in dental porcelain and their behavior during multiple firing. J Dent Res. 1996 Jul; 75(7): 1484-90. https://doi.org/10.1177/00220345960750070801

15. Felton DA, Kanoy BE, Bayne SC, Wirthman GP. Effect of in vivo crown margin discrepancies on periodontal health. J Prosthet Dent. 1991 Mar; 65(3): 357-64. DOI: https://dx.doi.org/10.1016/0022-3913(91)90225-L

16. Reich S, Schierz O. Chair-side generated posterior lithium disilicate crowns after 4 years. Clin Oral Investig. 2013 Sep; 17(7): 1765-72. https://doi.org/10.1007/s00784-012-0868-0

17. Bailey E. Contemporary views of dental practitioners on patient safety. Br Dent J. 2015 Dec; 219(11): 535-9. ht tps://doi.org/10.1038/sj.bdj.2015.920

18. Dourado A, Reis A. Sistemas adhesivos. RODYB [internet]. 2006 May-Ago; 1(2): 13-28. Disponible en: https:// www.rodyb.com/wp-content/uploads/2013/02/sistemas-adhesivos2.pdf

19. Pospiech P. All-ceramic crowns: Bonding or cementing? Clin Oral Investig. 2002 Dec; 6(4): 189-97. https://do i.org/10.1007/s00784-002-0183-2.

\section{Licencia Creative Commons CC BY 4.0}

Cómo citar: Pérez Gómez W, Pita Bejarano AM, Ramos Vargas CA, González Moncada J, Güiza Cristancho EH, Rodríguez Ciódaro A. Análisis de los eventos adversos en el área de rehabilitación oral de la Facultad de Odontología de la Pontificia Universidad Javeriana Bogotá. Univ Odontol. 2017 jul-dic; 36(77). https://doi.org/10.11144/Javeriana.uo36-77.aear 